\title{
Erratum
}

Arch Pharm Res Vol 28, No 4, 391-394 (2005)

\section{Synthesis of 3-Alkylthio-6-Allylthiopyridazine Derivatives and Their Antihepatocarcinoma Activity}

\section{Soon-Kyoung Kwon and Aree Moon}

College of Pharmacy, Duksung Women's University, Seoul 132-714, Korea

In Fig. 2A, * (statistical significance) must be inserted in $\mathrm{K} 6$ as shown.

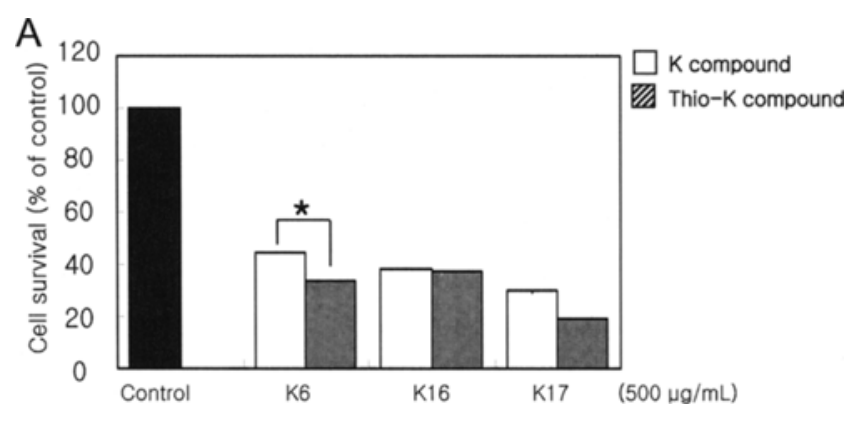

